\title{
Selective interspecific information use in the nest choice of solitary bees
}

\author{
Olli J. Loukola ${ }^{1,2,4, *}$, Elia Gatto ${ }^{3,4}$, Ana C. Híjar-Islas ${ }^{4}$ and Lars Chittka ${ }^{4,5}$ \\ ${ }^{1}$ University of Oulu, Ecology and Genetics Research Unit, PO Box 3000, FI-90014, Oulu, Finland \\ ${ }^{2}$ Botanical Museum, Biodiversity Unit, PO Box 3000, FI-90014, University of Oulu, Oulu, Finland \\ ${ }^{3}$ University of Padova, Department of General Psychology, 35100 Padova, Italy \\ ${ }^{4}$ Queen Mary University of London, Department of Biological and Experimental Psychology, \\ School of Biological and Chemical Sciences, London E1 4NS, United Kingdom \\ ${ }^{5}$ Wissenschaftskolleg zu Berlin, Institute for Advanced Study, Wallotstrasse 19, D-14193 Berlin, \\ Germany
}

Submitted: October 30, 2019. Final revision received: December 13, 2019.

Accepted: December 20, 2019

\begin{abstract}
Most of the studies on learning in bees have focused on the foraging context; we know little about the preferences and cognitive processes in nest-site selection, especially in solitary bees. The majority of the bee species are solitary and in contrast to eusocial bees, solitary bees' cognition and social information use have remained largely unstudied. Solitary cavity-nesting mason bees (Osmia spp.) are an ideal system to study interspecific information use in nest choice in the wild as many species share similar nesting requirements. Here, we show that the blue mason bee (O. caerulescens) and the orange-vented mason bee (O. leaiana) examine hallmarks of parasitization of the nests of red mason bees (O. bicornis) before deciding where to establish their own nests. They were also presented with contextual cues (geometric symbols) that could be linked to parasitization by observational learning. Subjects subsequently had the choice of nesting in a nest site marked by the symbol that matched, or did not match, the one seen at the parasitized or healthy nest. We show that the bees copied and rejected the symbol of the examined nest manipulated to exhibit successful and unsuccessful nesting, respectively. We conclude that solitary bees use interspecific information in their nest-site selection. In contrast with current theories of species coexistence, niche overlap between species may dynamically change depending on the observed success of surrounding individuals.
\end{abstract}

\section{Keywords}

Interspecific information use; nest choice; solitary bees; species coexistence

*) Corresponding author; e-mail: olli.loukola@oulu.fi 


\section{Introduction}

Animals typically reside in a variable and complex environment in which they have to exhibit the appropriate behaviour to secure access to food and nesting sites, or to avoid predation. In addition to gathering information about their environment individually by trial-and-error strategies, an individual can capitalize on cues left behind by others, or active observation of other animals' behaviour - typically conspecifics, but also from members of other species that share similar ecological requirements (Galef \& Giraldeau, 2001; Brown \& Laland, 2003; Freeberg, 2005; Leadbeater \& Chittka, 2007; Seppänen et al., 2007; Avarguès-Weber et al., 2013). Bees have become important study organisms to explore such learning from others, including the copying of flower choices from knowledgeable individuals (Leadbeater \& Chittka, 2007) and also learning object manipulation skills by observing skilled conspecifics (Alem et al., 2016; Loukola et al., 2017). These studies have typically been conducted on social species, even though the majority of the over 20,000 bee species are solitary (Michener, 2000).

An overwhelming majority of studies on learning in bees have focused on the foraging context, where bees associate flower cues with rewards (Giurfa, 2015; Leadbeater \& Dawson, 2017), so we know little about the preferences and cognitive processes in nest-site selection, especially in solitary bees (but see Seeley, 2010 for the social honeybees). An understanding of these processes is, however, crucial in the conservation of the many bee species now threatened not just by shortage of floral resources and emergent disease, but also by a lack of suitable nesting opportunities (MacIvor, 2017). In birds, migratory pied flycatchers (Ficedula hypoleuca) are sensitive to nest-site choices and performance of competing resident tits (Parus sp.): they copy nest-site features of tits with observably large clutch size, and reject features associated with small clutches (Forsman \& Seppänen, 2011; Seppänen et al., 2011; Loukola et al., 2013). However, it is not yet known whether this "Copy-the-successful"/"Reject-the-unsuccessful" strategy in the context of nestsite selection exists in other taxa, including in insects which include many species that, like birds, establish nests in which they provision their young.

An understanding of the effects of nesting information use in multispecies communities requires manipulative field experiments (Galef, 2004; Thornton \& Clutton-Brock, 2011) that have rarely been conducted (Reader \& Biro, 2010) due to the challenges of establishing controlled conditions in natural settings. Cavity nesting and solitary mason bees (Osmia spp.) are an ideal system to study various types of social information use in the wild. Several mason bees have overlapping resource needs and they are putative competitors, which is predicted to increase the probability of interspecific information use (Seppänen et al., 2007).

As many solitary bee species only have one generation per year and short seasonal activity periods, the nest-site decisions are crucial (Michener, 2000). Brood cell parasitism is common in cavity-nesting megachilid bees and involves high fitness costs (Krombein, 1967). Several generalist parasite species using cavitynesting bees as hosts leave an observable exit hole when leaving the host nest 
(Krombein, 1967; Strohm, 2011). Thus, natural selection should favour bees that recognize the hallmarks of nest healthiness and prefer nesting near healthy nests and avoid proximity to parasitized nests of others or reject abstract contextual cues that are linked to parasitization (Boyd \& Richerson, 1985; Henrich \& Gil-White, 2001; Laland, 2004; Sarabian et al., 2018; Litman, 2019). Here we studied with a field experiment whether blue and orange-vented mason bees (observers) use the nesting success of heterospecifics, red mason bees, as a basis of decision-making in nestsite selection. We used standardized artificial nests and simulated nesting success of red mason bees with randomly assigned treatments, which excludes the possibility that bees respond to some other correlate of red mason bee success or habitat quality factors (such as olfactory indicators of actual parasitization). In brief, we tested whether an observer bee's choice between two new, neutral nest-site feature preferences (choosing a nest site with one of two alternative geometric symbols) is affected by observing the apparent nesting success (nest has the appearance of being healthy or infected by brood cell parasites) of red mason bees (fig. 1, see Methods).

\section{Methods}

We examine the nest-site preferences of blue and orange-vented mason bees that often nest in close proximity in tunnels of dead wood (Michener, 2000). The observing individual is free to choose between nest sites differing only in the presence of alternative geometric symbols (fig. 1), after having inspected artificial nests (made to look like those of red mason bees) where signs of parasitization could be observed and associated with the geometric symbols (Forsman \& Seppänen, 2015). Importantly, these unnatural symbols have no ecological relevance. Obviously there is no conceivable benefit of copying/rejecting choices of a novel visual nest-site feature varying only in shape (triangle or circle). The experiment requires the alternative choices to be ecologically and visually neutral and evolutionarily novel, to isolate the behaviour of interest (copying/rejecting) from other ecological factors, from unconditioned responses and from an individual's history and experience. By using novel neutral symbols differing only in shape, we can expect that the bees do not have any innate or learned (via trial-and-error) preferences confounding the question of interest (Alatalo \& Mappes, 1996; Forsman et al., 2018).

\section{Setup of the experiments}

The experiments were conducted in parks and grasslands in Southeast England in Faversham, Harpenden in 2016 and London in 2016-2018 (Spitalfields City Farm (2016), Stepney City Farm and Tower Hamlets Cemetery Park (2016-2018)). Nestblock units (fig. 1; 20, 60 and 20 in 2016, 2017 and 2018, respectively) for observer bees were distributed a few weeks before they initiated nest building throughout the study area (around mid-April). The treatment (the perceived nesting success of early-nesting red mason bees) for each unit was randomized. In 2016, data was 


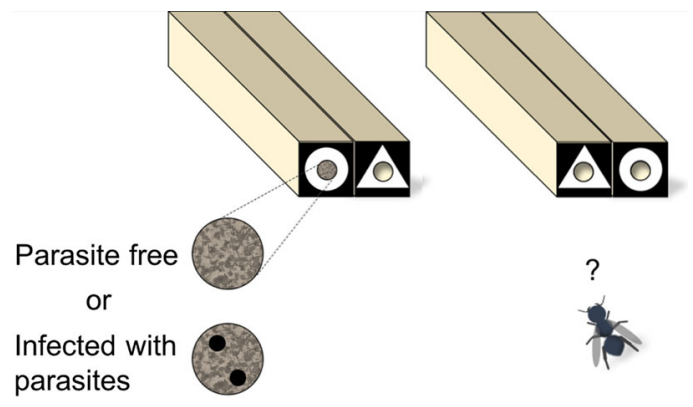

Figure 1. The experimental unit. The perceived success of earlier-nesting red mason bees was illustrated by using artificial nests made of mud that were divided into two treatments: (1) nest entrance plug was complete (successful $=$ no parasites) and (2) two small holes (diameter $0.8 \mathrm{~mm}$ ) were drilled through the plug (unsuccessful $=$ infected with parasites). A white symbol (circle or triangle) on a black background was then attached around the entrance hole of the artificial red mason bee nest block, while the alternative symbol was attached on an adjacent empty nest block immediately next to the red mason bee nest block. Two equivalent vacant nest blocks adjacent to each other, differing only by the type of symbol at their entrance were offered $20 \mathrm{~cm}$ from the artificial red mason bee nest block for bees nesting later. This design created an apparent choice of the earlier-nesting red mason bee towards one of the symbols (success associated with a symbol) in the first nest-block pair, while later-nesting bees had to choose between the two empty nest blocks $20 \mathrm{~cm}$ away from the red mason bee's nets-block pair. Replicate units were at least $20 \mathrm{~m}$ apart to make it less likely that bees were sampled repeatedly.

collected from 12 units located at: Faversham (four units in parasitized treatment), Harpenden (one and two units in healthy nest and parasitized treatments, respectively), Spitalfields City Farm (two units in healthy nest treatment) and Stepney City Farm (two and one units in healthy nest and parasitized treatments, respectively). In 2017, data were collected from 42 units located at: Harpenden (four units in parasitized treatment), Spitalfields City Farm (four and two units in healthy nest and parasitized treatments, respectively) and Tower Hamlets Cemetery Park (21 and 11 units in healthy nest and parasitized treatments, respectively). In 2018, data was collected from 13 units located at: Spitalfields City Farm (seven and two units in healthy nest and parasitized treatments, respectively) and Tower Hamlets Cemetery Park (two and two units in healthy nest and parasitized treatments, respectively). Each artificial red mason bee nest was built by using a wooden (dried birch, Betula pendula) block (length $12 \mathrm{~cm}$, height $2 \mathrm{~cm}$, width $2 \mathrm{~cm}$ ) with a drilled hole (diameter $8 \mathrm{~mm}$ ) sealed with a nest-entrance closure made of mud, mimicking the real appearance and substances used by red mason bees, which seal their nest entrance in this manner. The perceived nesting success of early-nesting red mason bees (flight period is from April to June) observed by later-nesting blue and orange-vented mason bees (flight period is from May to August) was manipulated by leaving the nest entrance completely sealed with mud or by drilling two small holes $(0.8 \mathrm{~mm}$ diameter) through the muddy closure. Complete muddy entrance closure indicated successful nesting of red mason bees from the same year, whereas 
two small holes in the closure plug indicated the entrance holes of the cleptoparasite Cacoxenus indagator or brood parasitoid Monodontomerus wasps, which in turn indicated unsuccessful nesting of red mason bees from a previous year (if parasitized by $C$. indagator, which has a one-year life-cycle; Coutin \& de Chenon, 1983) or the same year (if parasitized by Monodontomerus spp, which has only a three-week life-cycle; Eves, 1970). Cacoxenus indagator and Monodontomerus species are generalists and use both blue and orange-vented mason bees as hosts in our study areas (personal observations). A white symbol (circle or triangle) was then randomly selected (flip a coin) and painted on a black background around the entrance hole of the artificial red mason bee nest block, while the alternative symbol was attached on an adjacent empty nest block immediately next to the red mason bee nest block. Two equivalent vacant nest blocks adjacent to each other and differing only by the type of symbol at their entrance, were offered at $20 \mathrm{~cm}$ from the artificial red mason bee nest block for later-nesting bees. This display portrayed a red mason bee's choice between two novel, neutral alternative nest-site features (success associated with a feature), while observing bees had to choose between the two empty nest blocks further away from the first nest-block pair (fig. 1). Replicate units were at least $20 \mathrm{~m}$ apart to ensure independence. Nest-site characteristics (material and cavity size) and location (sun exposure) were designed to make the nest-site units as attractive as possible for the bees (MacIvor, 2017; pers. obs.) to ensure that bees would select one of the cavities within a unit, even in the proximity of an apparently parasitized nest.

\section{Data collection}

Data collection from 38 units was automated by using a light sensor-microcontroller-based system, on which the timing of each nest building was recorded. When the bee built its nest, the nest material blocked the sunlight and the light sensor registered this. An additional 29 units without an automated system were visually inspected every day; the bee's presence was recorded and the first nest-site choice was determined by the appearance of nest material. Also, the informationcollecting behaviour of female bees (scanning the simulated nest and symbol and inspecting the available cavities) was video-recorded at 16 different units (supplementary video S1), and the female bee's nesting behaviour (nest-entrance sealing) was video-recorded at one unit (supplementary video S2).

\section{Statistical analyses}

We used a single sample binomial test to determine if the observing bees' nest-site choices deviated from an expected proportion of 0.5. We used Bayesian generalized linear mixed models, package MCMCglmm (Hadfield, 2010) in R (version 3.2.5; R Core Team, 2016), to estimate the fixed effects of the treatment (simulated nesting success of red mason bee: parasite-free or infected with parasites), location of the red mason bee nest in the unit (from the observer's perspective: outer left, inner 


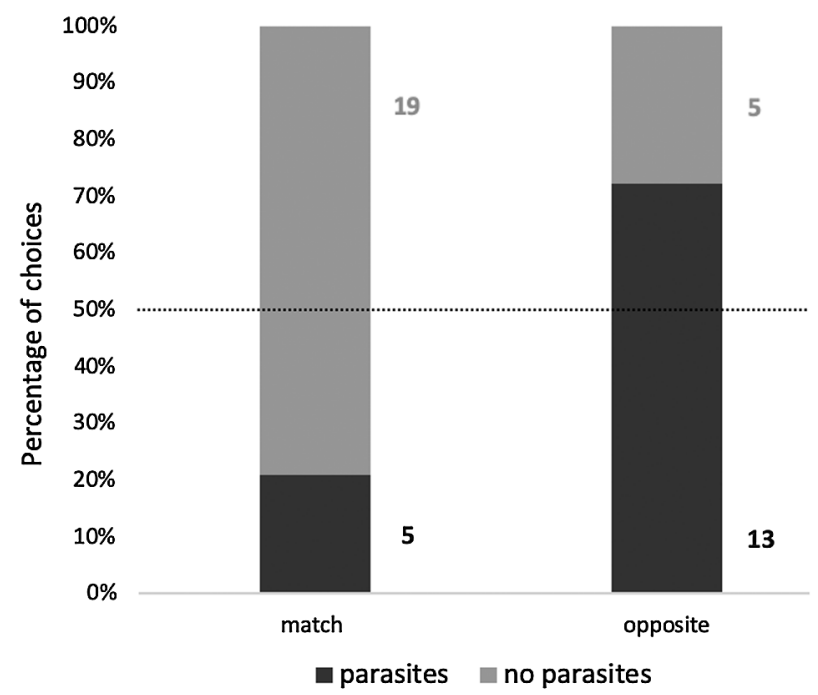

Figure 2. Nest-site feature choices of later-nesting bees. The bar on the left (match) denotes percentage of observing bees' nests in nest blocks with a symbol matching the symbol on the simulated red mason bee's nest block; the bar on the right (opposite) denotes percentage of observing bees' nests in nest blocks with a symbol opposing the symbol on the simulated red mason bee's nest block. The colours of the bars denote the treatment group (dark grey = parasitized red mason bee's nest; light grey $=$ healthy red mason bee's nest). The numbers on the right side of the bars denote the number of choices within a treatment group and the colour of the number denotes the treatment group. The dashed line denotes the null expectation of $50 \%$.

left, inner right or outer right), symbol attached to the red mason bee nest (circle or triangle) and the study year (2016, 2017 and 2018) for the nest-site choice of the observer bee (response variable, family binomial; symbol choice matching or opposing with the symbol on the artificial red mason bee nest) (fig. 2, table 1). In our model, we used the study site as random effect and a parameter-expanded prior that was minimally informative (Gelman, 2006) $(V=1, \boldsymbol{v}=0.002, \boldsymbol{\alpha} \times \boldsymbol{\mu}=0$, $\alpha \times V=1000$ ). We ran the model for 1400000 iterations with a burn-in of 400000 and sampling that produced 1000 estimates of the posterior distribution.

\section{Results and discussion}

In our experiments, each tested bee observed one artificial nest made to look like that of a red mason bee (fig. 1). Unlike red mason bees, which seal their nest holes with mud, blue and orange-vented mason bees use chewed leaves to seal their nest holes. The nest plugs of the two latter species are difficult to discriminate from each other. Thus, we pooled the nests of blue and orange-vented mason bees together. We measured a total of 42 choices of observing mason bees that chose one of the empty nests further away from the red mason bee nest-block pair (figs 1 and 2). In addition, 20 mason bees ( 8 and 12 in parasitized and healthy nest treatments, respectively) and five leaf-cutter bees (Megachile spp., two and three in parasitized and healthy 
Table 1.

Results of Bayesian generalized linear mixed-effects models (MCMCglmm) explaining the probability of the nest site choice of the observing bee. Study area is included as a random effect. The effective sample size for each variable is 1000 .

\begin{tabular}{lccc}
\hline Coefficient & Posterior mean & 95\% Credible interval & $p_{\text {MCMC }}$ \\
\hline Intercept & -7843 & -194000 to 188600 & 0.95 \\
Treatment (parasitized) & 446 & 62 to 875 & $0.008^{* *}$ \\
Red mason bee nest location (inner right) & 248 & -168 to 779 & 0.26 \\
Red mason bee nest location (outer left) & 193 & -200 to 695 & 0.36 \\
Red mason bee nest location (outer right) & -318 & -1169 to 283 & 0.35 \\
Red mason bee nest symbol (circle) & -6 & -524 to 454 & 0.99 \\
Red mason bee nest symbol (triangle) & 133 & -336 to 577 & 0.54 \\
Year & 4 & -93 to 96 & 0.95 \\
\hline
\end{tabular}

nest treatments, respectively) chose to nest in the empty nest block adjacent to the red mason bee nest block. We exclude these bees from the main analysis as our question of interest was focused on (and the experimental design was designed to study) only those bees that chose one of the empty nest blocks further away from the simulated nest. We analyzed the data for the bees that chose to build in the proximal nesting hole separately (supplementary text S1, supplementary fig. S1, supplementary table S1). Bees actively scanned the established artificial nest and the symbols and inspected the empty cavities before deciding where to establish their own nests (pers. obs., supplementary video S1). We found that the simulated nesting success of the red mason bee clearly affected the nest-site choice of the observing bees (fig. 2, table $1 ; p_{\text {MCMC }}=0.008$ ). As predicted, most observing bees (79, where chance expectation is $50 \%$, binomial test; observed $=19$, expected $=$ $12, z=2.65, p<0.004)$ copied the nest-site feature preference of an examined nest experimentally manipulated to exhibit successful nesting, whereas most bees presented with the hallmarks of parasitization rejected these in subsequent choices (72\%, binomial test; observed $=13$, expected $=9, z=1.65, p<0.05$; fig. 2). The location of the red mason bee's nest in the unit $\left(p_{\mathrm{MCMC}}>0.26\right)$, the symbol attached at the red mason bee's nest block $\left(p_{\mathrm{MCMC}}>0.54\right)$ or study year $\left(p_{\mathrm{MCMC}}=\right.$ 0.95 ) did not affect observer bees' nest-site choices (table 1). Our results from three years (2016-2018) and five study areas (see Methods) provide conclusive experimental evidence of solitary bees' response to heterospecific nesting success in their own nest-site selection. Presumably, they stored the symbol indicating nesting success (or failure) in working memory, and subsequently chose the matching one (if the symbol indicated success) or the non-matching one (if the symbol was linked to failure) (Chittka et al., 1997; Giurfa et al., 2001; Zhang et al., 2005; Raine \& Chittka, 2007). Solitary bees follow the same selective strategy as pied flycatchers when using interspecific information about nesting success (Loukola et al., 2013). This suggests that an active selective strategy to either copy or reject nest-site feature preferences of other species based on observed success, may be a common strategy 
in animals that share similar nesting requirements and habitats, and can have major consequences for niche overlap and partitioning. One of the central paradigms of evolutionary ecology, competition theory, postulates that two species with overlapping niches should be negatively impacted by the presence of the other due to competition, which in turn should lead to character displacement (Brown \& Wilson, 1956), niche segregation, and avoidance (MacArthur \& Levins, 1967; Schoener, 1974; Schluter, 2000). Decision-making strategies sensitive to perceived success of the observed individual make niche overlap a more behaviourally and ecologically dynamic phenomenon than assumed by competition theory. Niche overlap should increase through selective social information use towards the most successful individuals or in favourable environmental conditions that enhance the perceived fitness of the observed individuals. Conversely, active rejection of behavioural choices by poorly performing individuals can increase niche segregation and reduce costs of competition relative to the least successful individuals and in poor environmental conditions. Solitary bees do not return to their nest after they have provisioned and sealed it, and perish before having the opportunity to examine their nesting success (which will only be apparent the following spring). This suggests that "Copy-thesuccessful"/"Reject-the-unsuccessful" strategy (Laland, 2004; Loukola et al., 2013) could be an innate behaviour in this study.

Many observer bees (38\%) ignored or did not learn both the success/parasitization cue and the contextual symbols. Instead, despite the treatment, they built their nest in the nest block next to the simulated red mason bee nest. These bees probably used a different strategy, local enhancement (Heyes, 1994), in their decisionmaking, whereby they were attracted to the location of an established nest. Indeed, despite Osmia bees being solitary, many species are known to be gregarious, meaning that they prefer to build their nest next to that of other Osmia bees (Michener, 2000).

In contrast to eusocial bees, solitary bees' cognition and social information use has remained largely unstudied. To our knowledge, this is the first test of a solitary bee in a version of the match-to-sample task. Solitary bees' fitness depends on their own reproduction and not on their kin in a colony. Therefore, natural selection should favour making decisions based on the previous success of other individuals facing similar environmental pressures. Nesting suitability, foraging options, predation and parasitism can fluctuate spatially and temporarily within a community, and thus it makes sense for bees to glean information about nesting prospects from the success of other bees, even if these are members of different species.

\section{Limitations of the study}

This experimental design was originally developed to study the interspecific social information use between parid tits and pied flycatchers (Seppänen \& Forsman, 2007; Forsman \& Seppänen, 2011; Loukola et al., 2012, 2013). In these bird studies, flycatchers were restricted to choosing between the empty boxes of the nest-box pair further away from the tit nest-box pair. This is because breeding too close to a 
tit may result in fatally aggressive tit behaviour (Merilä \& Wiggins, 1995). We used the same design to allow direct comparison between cavity nesting birds and bees. However, Osmia bee females do not defend established nests. In addition, we used simulated nests and nothing was restricting the observer bees to choose the adjacent nest block next to the simulated red mason bee. It would have been technically easy to mark the individual bees. However, if we had done so near any of our sites, especially before they had begun nesting, they would likely have responded to this averse experience by not returning to the site. Finally, it would have been good to have full video coverage of the bees' decision-making process.

\section{Acknowledgements}

We thank Luigi Baciadonna, James Makinson, Joe Woodgate and HaDi Maboudi for helping in the field. We thank Janet and Tom Treasure, Stepney City Farm, Spitalfields City Farm, Tower Hamlets Cemetery Park who let us use their area as our study site. OJL is funded by the Jenny and Antti Wihuri foundation and Academy of Finland (no. 24302601) and the Chittka lab was funded by ERC Advanced Grant SpaceRadarPollinator and EPSRC grant Brains-on-Board. The manuscript was submitted to Animal Biology after initial evaluation in Peerage of Science (www.peerageofscience.org).

\section{Author contributions}

OJL designed the study and analyzed data; OJL, EG and ACHI collected the data. OJL and LC wrote the manuscript; all authors discussed the results and commented on the manuscript.

\section{Declaration of interests}

The authors declare no competing interests.

\section{Supplementary material}

Supplementary material is available online at: https://doi.org/10.6084/m9.figshare.11448315

\section{References}

Alatalo, R.V. \& Mappes, J. (1996) Tracking the evolution of warning signals. Nature, 382, 708-710.

Alem, S., Perry, C.J., Zhu, X., Loukola, O.J., Ingraham, T., Søvik, E. \& Chittka, L. (2016) Associative mechanisms allow for social learning and cultural transmission of string pulling in an insect. PLoS Biol., 14, e1002564. https://doi.org/10.1371/journal.pbio.1002564.

Avarguès-Weber, A., Dawson, E.H. \& Chittka, L. (2013) Mechanisms of social learning across species boundaries. J. Zool., 290, 1-11. 
Boyd, R. \& Richerson, P.J. (1985) Culture and the Evolutionary Process. University of Chicago Press, Chicago, IL, USA.

Brown, C. \& Laland, K.N. (2003) Social learning in fishes: a review. Fish Fish. (Oxf.), 4, 280-288.

Brown, W.L. \& Wilson, E.O. (1956) Character displacement. Syst. Zool., 5, 49-64.

Chittka, L., Gumbert, A. \& Kunze, J. (1997) Foraging dynamics of bumble bees: correlates of movements within and between plant species. Behav. Ecol., 8, 239-249.

Coutin, R. \& de Chenon, R.D. (1983) Biologie et comportement de Cacoxenus indagator Loew (Dipt., Drosophilidae) cleptoparasite d'Osmia cornuta Latr. (Hym., Megachilidae). Apidologie, 14, 233240.

Eves, J.D. (1970) Biology of Monodontomerus obscurus Westwood, a parasite of the alfalfa leafcutting bee, Megachile rotundata (Fabricius) (Hymenoptera: Torymidae: Megachilidae). Melanderia, 4, 1-18.

Forsman, J.T. \& Seppänen, J.-T. (2011) Learning what (not) to do: testing rejection and copying of simulated heterospecific behavioural traits. Anim. Behav., 81, 879-883.

Forsman, J.T., Seppänen, J.-T., Mönkkönen, M., Thomson, R.L., Kivelä, S.M., Krams, I. \& Loukola, O.J. (2018) Is it interspecific information use or aggression between putative competitors that steers the selection of nest-site characteristics? A reply to Slagsvold and Wiebe. J. Avian Biol., 49, jav-01558. https://doi.org/10.1111/jav.01558.

Freeberg, T.M. (2000) Culture and courtship in vertebrates: a review of social learning and transmission of courtship systems and mating patterns. Behav. Proc., 51, 177-192.

Galef, B.G. (2004) Approaches to the study of traditional behaviors of free-living animals. Anim. Learn. Behav., 32, 53-61.

Galef, B.G. \& Giraldeau, L.-A. (2001) Social influences on foraging in vertebrates: causal mechanisms and adaptive functions. Anim. Behav., 61, 3-15.

Gelman, A. (2006) Prior distributions for variance parameters in hierarchical models (comment on article by Browne and Draper). Bayesian Anal., 1, 515-534.

Giurfa, M. (2015) Learning and cognition in insects. Wiley Interdiscip. Rev. Cogn. Sci., 6, 383-395.

Giurfa, M., Zhang, S., Jenett, A., Menzel, R. \& Srinivasan, M.V. (2001) The concepts of 'sameness' and 'difference' in an insect. Nature, 410, 930-933.

Hadfield, J.D. (2010) MCMC methods for multi-response generalized linear mixed models: the MCMCglmm R package. J. Stat. Softw., 33, 1-22.

Henrich, J. \& Gil-White, F.J. (2001) The evolution of prestige: freely conferred deference as a mechanism for enhancing the benefits of cultural transmission. Evol. Hum. Behav., 22, 165-196.

Heyes, C.M. (1994) Social learning in animals: categories and mechanisms. Biol. Rev. Camb. Philos. Soc., 69, 207-231.

Krombein, K.V. (1967) Trap-Nesting Wasps and Bees. Smithsonian Press, Washington, DC, USA.

Laland, K.N. (2004) Social learning strategies. Anim. Learn. Behav., 32, 4-14.

Leadbeater, E. \& Chittka, L. (2007) Social learning in insects — from miniature brains to consensus building. Curr. Biol., 17, R703-R713.

Leadbeater, E. \& Dawson, E.H. (2017) A social insect perspective on the evolution of social learning mechanisms. Proc. Natl Acad. Sci. USA, 114, 7838-7845.

Litman, J.R. (2019) Under the radar: detection avoidance in brood parasitic bees. Philos. Trans. R. Soc. Lond. B Biol. Sci., 374, 20180196. https://doi.org/10.1098/rstb.2018.0196.

Loukola, O.J., Seppänen, J.-T. \& Forsman, J.T. (2012) Intraspecific social information use in the selection of nest site characteristics. Anim. Behav., 83, 629-633. 
Loukola, O.J., Seppänen, J.-T., Krams, I., Torvinen, S.S. \& Forsman, J.T. (2013) Observed fitness may affect niche overlap in competing species via selective social information use. Am Nat., 182, 474-483.

Loukola, O.J., Perry, C.J., Coscos, L. \& Chittka, L. (2017) Bumblebees show cognitive flexibility by improving on an observed complex behavior. Science, 355, 833-836.

MacArthur, R. \& Levins, R. (1967) The limiting similarity, convergence, and divergence of coexisting species. Am. Nat., 101, 377-385.

MacIvor, J.S. (2017) Cavity-nest boxes for solitary bees: a century of design and research. Apidologie, 48, 311-327.

Merilä, J. \& Wiggins, D.A. (1995) Interspecific competition for nest holes causes adult mortality in the collared flycatcher. The Condor, 97, 445-450.

Michener, C.D. (2000) The Bees of the World. Johns Hopkins University Press, Baltimore, MD, USA.

R Core Team (2016) R: a Language and Environment for Statistical Computing. R Foundation for Statistical Computing, Vienna, Austria.

Raine, N.E. \& Chittka, L. (2007) Flower constancy and memory dynamics in bumblebees (Hymenoptera: Apidae: Bombus). Entomol. Gen., 29, 179-199.

Reader, S.M. \& Biro, D. (2010) Experimental identification of social learning in wild animals. Learn. Behav., 38, 265-283.

Sarabian, C., Curtis, V. \& McMullan, R. (2018) Evolution of pathogen and parasite avoidance behaviours. Philos. Trans. R. Soc. Lond. B Biol. Sci., 373, 20170256. https://doi.org/10.1098/rstb. 2017.0256.

Schluter, D. (2000) The Ecology of Adaptive Radiation. Oxford University Press, Oxford, UK.

Schoener, T.W. (1974) Resource partitioning in ecological communities. Science, 185, 27-39.

Seeley, T.D. (2010) Honeybee Democracy. Princeton University Press, Princeton, NJ, USA.

Seppänen, J.-T. \& Forsman, J.T. (2007) Interspecific social learning: novel preference can be acquired from a competing species. Curr. Biol., 17, 1248-1252.

Seppänen, J.-T., Forsman, J.T., Mönkkönen, M. \& Thomson, R.L. (2007) Social information use is a process across time, space, and ecology, reaching heterospecifics. Ecology, 88, 1622-1633.

Seppänen, J.-T., Forsmanm, J.T., Mönkkönen, M., Krams, I. \& Salmi, T. (2011) New behavioural trait adopted or rejected by observing heterospecific tutor fitness. Proc. R. Soc. Lond. B Biol. Sci., 278, 1736-1741.

Strohm, E. (2011) How can cleptoparasitic drosophilid flies emerge from the closed brood cells of the red Mason bee? Physiol. Entomol., 36, 77-83.

Thornton, A. \& Clutton-Brock, T. (2011) Social learning and the development of individual and group behaviour in mammal societies. Philos. Trans. R. Soc. Lond. B Biol. Sci., 366, 978-987.

Zhang, S., Bock, F., Si, A., Tautz, J. \& Srinivasan, M.V. (2005) Visual working memory in decision making by honey bees. Proc. Natl Acad. Sci. USA, 102, 5250-5255. 